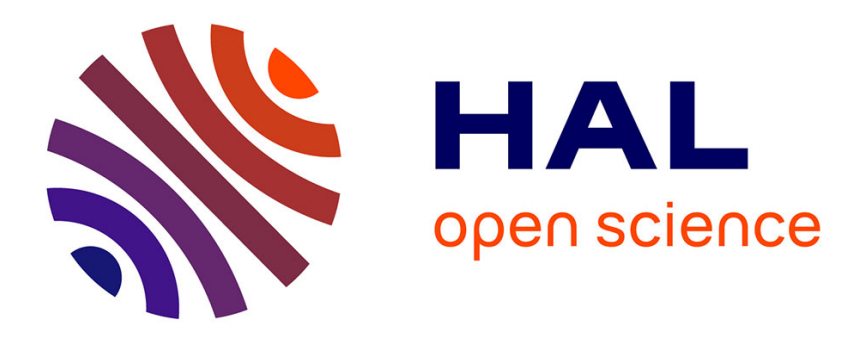

\title{
Recherches sur l'aimantation aux très basses températures
}

Pierre Weiss, H. Kamerlingh Onnes

\section{To cite this version:}

Pierre Weiss, H. Kamerlingh Onnes. Recherches sur l'aimantation aux très basses températures. J. Phys. Theor. Appl., 1910, 9 (1), pp.555-584. 10.1051/jphystap:019100090055500 . jpa-00241574

\section{HAL Id: jpa-00241574 https://hal.science/jpa-00241574}

Submitted on 1 Jan 1910

HAL is a multi-disciplinary open access archive for the deposit and dissemination of scientific research documents, whether they are published or not. The documents may come from teaching and research institutions in France or abroad, or from public or private research centers.
L'archive ouverte pluridisciplinaire HAL, est destinée au dépôt et à la diffusion de documents scientifiques de niveau recherche, publiés ou non, émanant des établissements d'enseignement et de recherche français ou étrangers, des laboratoires publics ou privés. 


\section{REGHERGHES SUR L'AIMANTATION AUX TRÈS BASSES TEMPÉRATURES;}

Par MM. Pierre WEISS et H. KAMERLINGH ONNES.

\section{§ 1. - But des recherches, résultats.}

a) Introduction. - Depuis que la théorie cinétique du magnétisme de Langevin (1) a pu être étendue, par l'hypothèse du champ moléculaire $\left(^{2}\right)$, à l'ensemble des faits du ferromagnétisme, il y a grand intérêt à confronter les conséquences de la théorie avec les données de l'expérience. L.es premiers résultats de cette comparaison ont été des plus encourageants en ce que, par certains côtés, des concordances remarquables ont été notées. C'est ainsi que la courbe calculée pour la variation de l'intensité d'aimantation à saturation en fonction de la température s'accorde très bien avec les observations sur la magnétite pour les températures au-dessus de la température ordinaire. La loi trouvée par l'hypothèse du champ moléculaire pour la susceptibilité au-dessus du point de Curie $\left(^{\mathbf{3}}\right)$, est vérifiée exactement dans des intervalles de plusieurs centaines de degrés par les expériences de Curie et par des mesures inédites. Les valeurs de la discontinuité de la chaleur spécifique au point de Curie calculée d'après les données magnétiques et observées ont été trouvées concordantes. Mais d'autres expériences s'accordent moins bien avec la théorie. La fig. A, dans laquelle a été représentée en trait plein la courbe $(a)$ théorique de la variation de l'aimantation à saturation en fonction de la température avec les points donnés par l'expérience pour la magnétite contient aussi la courbe (b) analogue trouvée expérimentalement pour le nickel ('). Elle a été dessinée à une échelle telle que la concordance soit aussi bonne que possible au point de Curie $\theta$. (On voit que, contrairement à ce qui se

(1) Laxgevin, Ann. Chim. Phys., 8c série, t. I', p. $70 ; 1905$.

(2) P. Weiss, J. de Phys., $4^{\circ}$ série, t. VI, p. $661 ; 1907$.

(3) C'est ainsi que nous appellerons dans ce travail la température de perte du ferromagnétisme spontané. Il n'y a là aucune contradiction avec l'idée de température de transformation fonction du champ énoncée par Curie, la température de perle du ferromagnétisme spontané étant celle que l'on détermine en faisant tendre le champ vers zéro.

(4) D'après des expériences préliminaires. Les mesures définitives à haute température sont en voie d'exécution. 
passe pour la magnétite, ici l'écart entre la théorie et l'expérience se manifeste progressivement dans toute l'étendue de la courbe. Le fer et le cobalt se comportent à peu près comme le nickel. Dans cet état de cause, le champ moléculaire prend le caractère d'une hypothèse de travail, les succès partiels garantissant qu'elle contient une part de vérité et les discordances indiquant la voie dans laquelle il convient de rechercher les retouches ou les généralisations conservant ce qu'elle a d'essentiel.

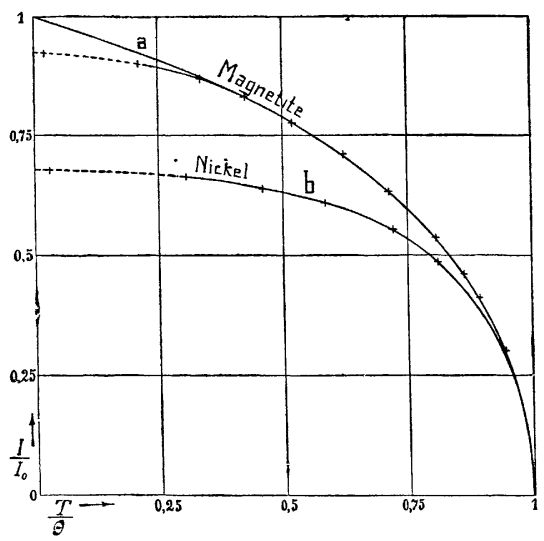

FIG. A.

Il ne semble pas que les remaniements puissent s'attaquer au rôle de l'énergie cinétique, s'opposant à l'orientation par le champ, ou à l'emploi fait de la loi de répartition de Maxwell-Boltzmann. Outre le caractère fondamental de ces notions, la facilité avec laquelle elles représentent la susceptibilité inversement proportionnelle à la température absolue des paramagnétiques, l'une des lois les mieux établies par l'expérience pour un certain nombre de corps, impose leur conservation. H. du Bois et Honda viennent de montrer, il est vrai, dans un remarquable travail sur l'aimantation des corps simples, résumé dans une note récente( $\left.{ }^{1}\right)$, que cette dépendance thermique de la susceptibilité n'est pas générale et qu'il se rencontre aussi le paramagnétisme indépendant de la température ou croissant avec elle. Loin d'imposer le rejet de ces notions, l'expérience vient ici

(1) Acad. Amsterdam, p. 666, janvier 1910. 
rappeler qu'elles ne contiennent pas tous les éléments du magnétisme. Il reste à réviser notamment l'hypothèse de Langevin de l'invariabilité, ou de la quasi-invariabilité, du moment magnétique de la molécule et celle de la natur e des actions mutuelles exprimées jusqu'à présent par le champ moléculaire. Or, pour le contrôle de l'une et l'autre hypothèse, les recherches à très basses températures sont particulièrement précieuses, car ce n'est qu'au zéro absolu que, le raccourci provoqué par l'agitation thermique ayant disparu, l'intensité d'aimantation donne directement la somme de ces moments magnétiques moléculaires, et c'est aussi aux basses températures qu'on doit s'attendre à trouver les actions mutuelles les plus vives, par suite de l'extrême réduction des distances moléculaires.

b) Ferromagnétiques. - Nous nous sommes donc proposé de continuer les courbes de variation de l'aimantation à saturation, pour les trois métaux ferromagnétiques et la magnétite, jusque dans le voisinage du zéro absolu. En employant les méthodes et dispositifs ( ${ }^{1}$ ) appropriés aux mesures de longue durée aux températures constantes, accessibles par l'hydrogène liquide nous avons pu opèrer à $20^{\circ}, 4 \mathrm{~K}$. par l'ébullition dans le voisinage de la pression atmosphérique et à $14^{\circ}, 0 \mathrm{~K}$. par la congélation $\left(^{2}\right)$. Ces quelques degrés séparant la température de l'observation du zéro absolu sont une fraction si petite de l'intervalle du zéro alssolu au point de Curie qui, mème pour le nickel, compte environ 648 degrés que, eu égard à l'allure des courbes, la valeur de l'aimantation à saturation au zéro absolu peut être considérée comme déterminée. Ceci sous la réserve que le phénomène ne prend pas une physionomie nouvelle dans l’intervalle d'extrapolation.

L'objet de la mesure étant l'aimantation à saturation, il semblait indiqué de diriger les expériences vers la connaissance de l'aimantation dans les champs élevés avec détermination de la loi d'appr che. Mais la méthode que nous avons choisie pour les mesures magnétiques et qui consiste à mesurer le couple maximum exercé sur un ellipsoïde de la subsıance far un champ d'orientation variable (voir $\S 2$ ), se prêtait moins bien à cette détermination de la loi d'approche pour des raisons qui seront exposées, qu'à la comparaison des

(1) H. Kanerlixgh Onnes, Corrmunic. Physical Labor., n 94 f ; Leiden, 1906.

(?) La lettre $\mathrm{K}$ (Kelvin) indique la température absolue. La réduction à l'échelle thermodynamique est faite au moyen des isothermes de l'hélium aux basses températures. Voir Kamerlingh Onnes, Physical Labor., n $102 b$; Leiden, 1908. 
aimantations que prend une substance à diverses températures pour une même valeurdu champ. Les renseignements sur la loi d'approche ont donc été demandés à une étude indépendante (1). Elle a donné, pour les écarts entre les aimantations dans un champ de 10.000 gauss et la valeur limite:

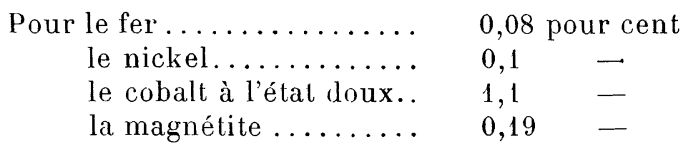

Pour ces substances, le cohalt excepté, la loi d'approche est hyperbolique, de sorte qu'à 20.000 gauss, champ atteint dans nos expériences, ces écarts sont réduits de moitié. Les séries de mesures à champ variable faites par la méthode de l'ellipsoïde à basse température et à la température ordinaire ont permis non de contrôler la loi d'approche, mais de montrer qu'elle n'est pas essentiellement différente aux deux températures et qu'il ne se manifeste pas, comme on aurait pu le supposer, aux basses températures, une dureté magnétique extrême provenant du blocage des molécules les unes par les autres.

Procédant par mesures relatives, on a donc compars, pour plusieurs valeurs élevées du champ, l'aimantation à la température ordinaire et à basse température et constaté que ce rapport est sensiblement indépendant du champ. On a trouvé ainsi pour le rapport entre l'intensité d'aimantation à $20^{\circ}, 3 \mathrm{~K}$. et à la température ordinaire, la correction de la dilatation entre les deux températures n'étant pas faite (voir p. วั66, note 2):

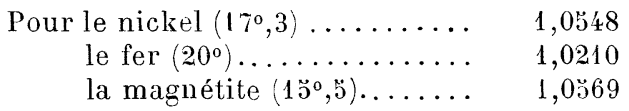

Le nombre entre parenthèses précise la température ordinaire. Il est dit au $\S$ a pourquoi l'exjérience n'a pas abouti pour le cobalt. L'exactitude probable de ces résultats est difficile à évaluer. La technique expérimentale, telle qu'elle a été élaborée dans tous ses détails, permettrait d'atteindre une précision de un millième ou même plus giande. Mais, en présence des perturbations qui se

(1) P.WeIss, Archives des Sc.phys. et nat., 4 pér., t. XXIX, p. 173̈; 1910 ; et J. de Phys., 'e série, t. IX. p. 373; 1910. 
AIMANTATION AUX TRÉS BASSES TEMPÉRATURES 559 sont manifestées pour le cobalt, il serait imprudent, semble-t-il, d'affirmer, pour la magnétite surtout, une exactitude supérieure

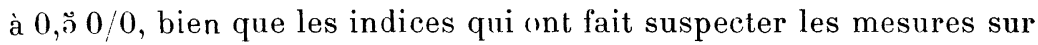
le cobalt aient presque totalement fait défaut pour les autres corps et que le cobalt, par l'ensemble de ses propriétés et notamment par son extrême dureté magnétique, occupe nettement une place à part. En mettant en évidence ces causes d'incertitude, nos expériences montrent la marche à suivre pour arriver, quand on le voudra, à une plus haute précision. Ia précision actuelle est d'ailleurs suffisante dans bien des cas.

Pour le fer et la magnétite, des mesures ont été faites jusqu'à $14^{\circ}, 0 \mathrm{~K}$. La variation de l'aimantation de $20^{\circ}, 3 \mathrm{~K}$. à $14^{\circ}, 0 \mathrm{~K}$. est trop faible pour être exprimée numériquement avec certitude. Ces expériences étendent donc seulement jusqu'à $1^{\circ}, 0 \mathrm{~K}$. l’intervalle de température dans lequel l'abaissement de l'énergie cinétique et le rapprochement des molécules ne provoque aucun phénomène nouveau. Les parties nouvelles des courbes du nickel et de la magnétite sont figurées en pointillé dans la fig. A.

Le cas de la magnétite est particulièrement intéressant par la concordance exacte de la théorie et de l'expérience dans la plus grande partie de l'intervalle du zéro absolu au point de Curie et l'écart qui se manifeste aux basses températures seulement. La théorie lonne en effet pour le rapport des aimantations 1,139 au lieu de 1,0ङ7 donné ci-dessus.

Cette divergence est appuyée par des expériences antérieures faites sur quatre échantillons de magnétite différents, deux provenant de cristaux naturels, le troisième d'un cristal naturèl refondu et le quatrième de magnétite artificielle, qui ont donné pour le rapport de l'aimantation dans la neige carbonique $-79^{\circ} \mathrm{C}$. et à la température ordinaire :

\begin{tabular}{|c|c|c|}
\hline & 1033 & Température ordinaire $16^{\circ}$ \\
\hline & 1042 & $23^{\circ}, 2$ \\
\hline & 1043 & $24^{\circ}, 0$ \\
\hline & $103 \pi$ & $21^{\circ}, 5$ \\
\hline Moyenne & 1039 & $21^{\circ}, 2$ \\
\hline
\end{tabular}

alors que la théorie donne, pour le même intervalle de température : 1.033

Il semble donc qu'il y ait analogie avec les phénomènes de la com- 
pressibilité et de la dilatation thermiques pour lesquels la théorie de van der Waals, et la loi des états correspondants thermiques se vérifient bien en général dans le voisinage du point critique, tandis qu'aux faibles valeurs de la température réduite la molécule et les lois d'altraction schématisées ne s'adaptent plus suffisamment aux faits et les propriétés individuelles des molécules réelles se manifestent.

L'hypothèse de l'invariabilité fondamentale de l'aimant moléculaire aurait reçu un appui particulièrement décisif si, comme cela peut être suggéré par les intensités à saturation régulièrement espacées des trois métaux, il y avait des rapports simples entre les moments magnétiques des atomes. Le tableau suivant :

\begin{tabular}{|c|c|c|c|c|c|c|}
\hline \multirow[b]{2}{*}{$\mathrm{Ni} . .}$. & \multicolumn{2}{|c|}{$\begin{array}{l}\text { Satur. spécif. } \\
\text { aux temp. ( ) }\end{array}$} & \multirow{2}{*}{$\begin{array}{c}\text { Coeff. d'amplific. } \\
\text { à très basse } \\
\text { temp. } \\
1,0 \mathbf{J}^{\prime} \mathbf{4} 8\end{array}$} & \multirow{2}{*}{ 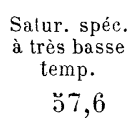 } & \multirow{2}{*}{$\begin{array}{l}\text { Masse atom. } \\
\text { ou } \frac{1}{3} \text { moléc. } \\
58,7\end{array}$} & \multirow{2}{*}{$\begin{array}{c}\text { Moment } \\
\text { de l'atome- } \\
\text { gramme } \\
3381\end{array}$} \\
\hline & 54,6 & $\left(17^{\circ}\right)$ & & & & \\
\hline & 162 & $\left(17^{\circ}\right)$ & 1,01 & 163,6 & 59 & 9650 \\
\hline & 217 & $\left(20^{\circ}\right)$ & 1,021 & 221,6 & 56 & 12410 \\
\hline $\mathrm{FeO}^{\frac{4}{3}}$. & 90,75 & $\left(15^{\circ}, 8\right)$ & 1,057 & 95,9 & $7 \pi, 33$ & 7417 \\
\hline
\end{tabular}

dans lequel les nombres de la première colonne sont empruntés à un travail déjà cité $\left.{ }^{1}\right)$ et où l'accroissement relatif pour le cobalt a été estimé par analogie avec le fer et le nickel montre qu'il n'en est rien $\left({ }^{2}\right)$. Mais, bien entendu, si dans ce cas la démonstration de la signification primordiale de cette quantité nous échappe, rien n'autorise une conclusion contraire.

Quand nous considérons l'ensemble de nos mesures nous sommes disposés à conserver l'hỵpothèse de l'invariabilité au moins approximative de l'atome magnétique en fonction de la température. Il y avait en effet des raisons pour la mettre en question aux très basses températures en admettant même qu'elle soit prouvée dans les autres circonstances. La résistance électrique des métaux, la phosphorescence des sulfures, l'absorption de la lumière par les sels des terres rares avec ou sans champ magnétique, tous ces phénomènes ont aux très basses températures des propriétés que l'on peut essayer d'expliquer en y faisant intervenir les forces exercées par la matière pondérable sur les électrons; ces forces jouent un rôle prépondérant quand la température tombe à celle de l'hydrogène liquide. On peut

(1) P. Weiss, Arch. Sc. phys. et nat. et J. de Phys., 1910.

(2) Le correction de la dilatation thermique (voir p. 566, note 2) ne modifiera pas sensiblement les rapports des nombres de la dernière colonne. 
AIMANTATION AUX TRES BASSES TEMPÉRATURES 561 leur attribuer en particulier l'importante diminution du nombre des électrons conduisant le courant électrique dans les métaux qui sont en quelque sorte gelés sur les atomes par l'abaissement de la température $(1)$.

On aurait pu imaginer que les mouvements des électrons du magnétisme, invariables ou à peu près aux autres températures, commençassent, eux aussi, à subir des changements importants au bas de l'échelle thermométrique. Mais puisque rien dans nos résultats n'invite à faire cette hypothèse on sera tenté plutôt de conclure que les électrons du magnétisme sont différents de ceux qui produisent les autres phénomènes.

c) Vanadium, chrome, manganèse. - On s'est demandé souvent s'il y a une différence de propriétés irréductible entre les métaux ferromagnétiques de la famille du fer etles métaux paramagnétiques de la même famille ou s'il suffirait d'abaisser suffisamment la température de ces derniers pour atteindre un point de Curie situé très bas. Ch.-Ed. Guillaume $\left({ }^{2}\right)$ dit à propos des alliages de $\mathrm{Mn}, \mathrm{Al}, \mathrm{Cu}$ et de $\mathrm{Mn}, \mathrm{Sn}, \mathrm{Cu}$ de Heusler qui sont ferromagnétiques: la raison peut en être trouvée dans le fait que l'aluminium ou l'étain, combinés avec le manganèse, métal du groupe magnétique, relèvent sa température de transformation, située très bas, conformément à une hypothèse formulée déjà par Faraday. On constate en effet que $\mathrm{Al}$ et $\mathrm{Sn}$ relèvent les points de fusion de plusieurs alliages qu'ils forment avec d'autres métaux (séries Al-Au, Al-Sb, Na-Sn), et semblent posséder, d'une façon tout à fait générale, la propriété de relever les températures de transformation.

Nous devions donc nous attendre à trouver pour le vanadium, le chrome et le manganèse à très basse température, soit des phénomènes ferromagnétiques (non-proportionalité de l'aimantation au champ, saturation, hystérèse), soit un paramagnétisme considérablement amplifié conformément à la loi de Curie. A la température de l'hydrogène solide la susceptibilité devait être, d'après cetteloi, environ 20 fois plus forte qu'à la température ordinaire $\left({ }^{3}\right)$. Nous n'avions

(1) C.. H. Kamerlingh Onnes, Communic. Physical Labor., Suppl. no 9, p. 27 ; Leiden, 1904; et P. Lenard, H. Kamerlivgh Onnes and W.-E. PaUli, id., nº 111, p. 3, note 2 ; Leiden, 1909.

(2) Actes de la Soc. helvét. des sc. nat., vol. I, p. $88 ; 1907$.

$\left.{ }^{3}\right)$ Une supposition analogue a été le point de départ de recherches faites par

H. Kamerlingh Onnes et A. Perrier, en relation étroite avec le présent travail.

Ces recherches ont été entreprises en même temps que ce travail et seront 
pas, en effet, connaissance des résultats publiés depuis par H. du Bois et Honda (') desquels il résulte que le paramagnétisme inversement proportionnel à la température absolue n'est qu'un des cas possibles. Pour se faire une idée de l'ordre de grandeur des phénomènes attendus on peut imaginer que le fer $\gamma$ paramagnétique continue à exister à $14^{\circ} \mathrm{K}$. avec la même constante de Curie (produit de la susceptibilité spécifique par la température absolue). On trouve alors, pour son aimantation dans un champ de 20.000 gauss, environ 400 . Gebhardt $\left(^{*}\right)$ a déterminé récemment la susceptibilité du manganèse à la température ordinaire et trouve $\mathrm{K}=322 \cdot 1^{-6}$ (densité 6,4 ). Le même calcul donne pour ce corps dans les mêmes conditions une intensité d'aimantation égale à 134. Et, coınme l'appareil dont nous nous sommes servi prend des déviations proportionnelles au carré de l'aimantation, on trouverait des déviations 18 fois, pour le inanganèse, et, dans le cas du fer $\gamma, 160$ fois plus petites que celle donnée par le fer à la température ordinaire, qui était de 100 centimètres, c'est-à-dire triès aisément observables.

Or en mettant successivement dans l'appareildes ellipsoïdes grossièrement taillés dans du vanadium de Moissan, du chrome et du manganèse de Goldschmidt, on n'a pas observé les changements attendus. La déviation est restée, dans tous les cas, à la température de l'hydrogène liquide sous pression atmosphérique et à celle de sa solidification, ce qu'elle était à la température ordinaire, c'est-à-dire les quelques dixièmes de millimètre dus au magnétisme propre du support. Il n'y avait donc pas de ferromagnétisme, et il était nécessaire de choisir, pour ces corps, entre les deux hypothèses suivantes: ou ces corps présentaient un paramagnétisme d'un type nouveau ou ils étaient diamagnétiques comme le cuivre dont la plupart des sels sont paramagnétiques. Le travail de du Bois et Honda, dans lequel ces trois métaux figurent parmi ceux qui ont un paramagnétisme constant ou croissant avec la température, montre que c'est la première supposition qui est exacte. L'exemple du cuivre nous

publiées prochainement. En se servant de la méthode du couple praximum et de celle de l'ascension hydrostatique on a déterminé l'aimantation de l'oxygène liquide à diverses températures et celle de l’oxygène solide aux températures de l'ébullition et de la solidification de l'hydrogène. L'accroissement de l'aimantation aux basses températures a été trouvée très grande, bien qu inférieure a celle qui était attendue; la courbe de variation thermique indique un écart caractérisé de la loi de Curie.

(1) H. DU Bors et Honda, loc. cit.

(2) GebH trdt, Inaug. Dissert. Marburg, 1909. 
AIMANTATION AUX TRÉS BASSES TEMPÉRATURES 563 a fait considérer l'autre hypothèse comme plausible. On pouvait penser en effet que le paramagnétisme, généralement attribué au manganèse métallique, est le résultat de la présence de ses oxydes fortement magnétiques ou de putites quantités de fer. Pour mettre cette hypothèse à l'épreuve, nous avons préparé du manganèse très pur en partant du chlorure pur de Merck vérifié sans fer, que nous avons électrolysé avec une cathode de mercure distillé et une anode de platine rhodié à 40 0/0 qui n'est pas attaquée par l'ion chlore.

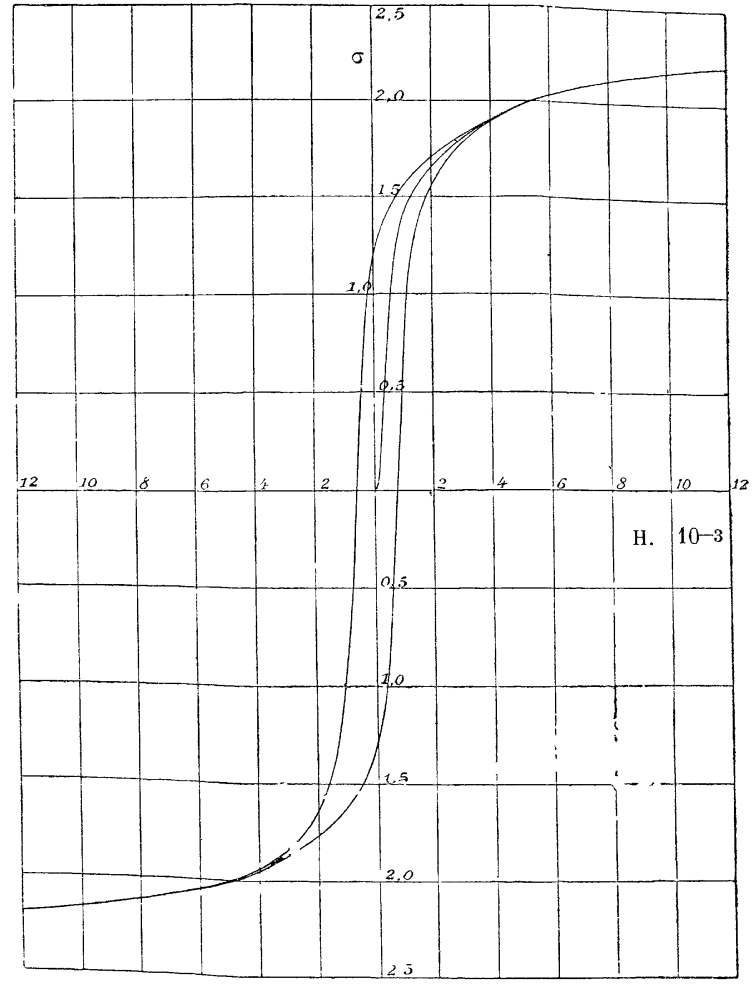

FIG. B.

L'amalgame ainsi obtenu a été décomposé par la chaleur dans un courant d'hydrogène pur et sec. On obtient áinsi une poudre grise, agglomérée sous forme d'un bâtonnet assez résistant quand l'amalgame a été moulé dans un tube de verre. Ce bâtonnet a été trouvé 
paramagnétique. Il en a été de mème pour un tube de verre rempli de manganèse pulvérulent.

Ce même manganèse a ensuite été fondu au four électrique à résistance dans une nacelle en magnésie et dans une atmosphère d'hydrogène. On a obtenu ainsi un lingot recouvert d'une croûte légèrement oxydée Après avoir reconnu qu'il était impossible de tailler le métal avec de la poudre de quartz dont il a exactement la dureté et ne pouvant employer l'émeri, magnétique, on l'a débarrassé de sa croûte impure en le tournant sous forme d'un petit cylindreavec un outil en diamant.

Ce cylindre a manifesté des propriétés ferromagnétiques. La fig. B donne un cycle d'hystérèse de cette substance. La valeur maxima de l'aimantation spécifique $\sigma$ est environ $\mathbf{1 0 0}$ fois plus faible que celle du fer et le champ coercitif est de 670 gauss, c'est-à-dire environ dix fois plus fortque celui d'un bon acier à aimants permanents. Cette étrange substance semble en outre avoir des propriétés magnétocristallines marquées. Entre les pôles d'un aimant le barreau était fortement attiré par les pôles tout en se plaçant perpendiculairement au champ.

Le même manganèse, également pur, peut donc exister sous deux états: paramagnétique et ferromagnétique. On peut déduire des expériences de Gebhardt une susceptibilité spécifique environ cinq fois plus forte que celle de du Bois. Si la poudre de manganèse de Gebhardt n'était pas impure ou oxydée, il se pourrait donc qu'il y eût deux états paramagnétiques.

Quant au ferromagnétisme du manganèse, il a déjà été observé par Seckelson $\left({ }^{1}\right)$ sur du manganèse électrolytique déposé, à la température de $100^{\circ}$, du chlorure sur un fil de platine et sur un lingot préparé par Bunsen à partir du tluorure. Les observations assez vagues qu'il donne sur son aimantation ne semblent pas incompatibles avec nos mesures.

Nous avons contrôlé l'absence de magnétisme intense dans le vanadium, le chrome et le manganèse à basse température par une expérience plus directe. (In a mis les ellipsoïdes des trois substances dans un tube Dewar étroit sans argenture placé lui-même dans un deuxième tube Dewar destiné à recevoir de l'air liquide et l'on a cherché à quelle distance ils étaient attirés du fond dı vase aux pôles

(1) Seckelson, Wied. Ann., t. LXVII, p. 37 ; 1899. 
AIMANTATION ALX TRÉS BASSES TEMPÉRATURES 565 de l'aimant. Cette expérience a été faite avec les tubes Dewar vides et dans l'hydrogène liquide. On a trouvé ainsi :

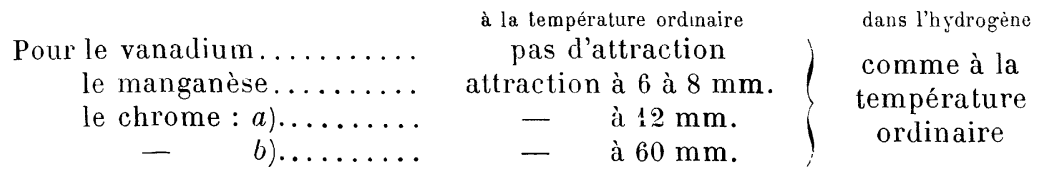

Le chrome $b$, qui contient probablement une paillette de fer, a été rejeté.

Cristal de sulfate ferreux. $\quad \begin{aligned} & \text { à la température ordinaire } \\ & \text { attraction à } 25 \mathrm{~mm} .\end{aligned}\left\{\begin{array}{c}\text { dans l'hydrogène } \\ \text { attiré presque depuis } \\ \text { le socle de l'aimant. }\end{array}\right.$

Ainsi la faible aimantation des trois métaux a été trouvée sensiblement invariable, tandis que le sulfate de fer a manifesté un accroissement énorme des propriétés magnétiques.

Cette expérience est bien faite pour montrer la différence caractéristique des deux groupes de corps et est un exemple typique de la portée que peuvent avoir les expériences les plus simples dans la région inexplorée des très basses températures.

\section{§ 2. - MÉthode et appareils.}

a) Discussion de la méthode du couple maximum. - L'intensité d'aimantation a été mesurée en déterminant le couple exercé sur un ellipsoïde de révolution allongé de la substance à étudier par le champ d'un électro-aimant faisant un angle variable avec le grand axe de cet ellipsoïde. Le couple a pour expression :

$$
\mathbf{M}=\left(\mathbf{N}_{1}-\mathbf{N}_{2}\right) \mathbf{I}^{2} v \sin \varphi \cos \varphi,
$$

où $\mathrm{N}_{1}$ et $\mathrm{N}_{2}$ sont les coefficients démagnétisants de l'ellipsoïde, I l'intensité d'aimantation de la substance, $v$ le volume et $\rho$ l'angle de I avec le grand axe de l'ellipsoïde. Ce couple est maximum :

$$
\mathrm{M}=\frac{\mathrm{N}_{1}-\mathrm{N}_{2}}{2} \cdot \mathrm{I}^{2} v
$$

pour $\varphi=45^{\circ}$. Il n'est donc nécessaire, pour mesurer I, de connaître ni la valeur du champ, ni l'azimut du champ pour lequel ce maximum est obtenu. Pour mettre cette méthode en œuvre, on suspend l'ellipsoïde à un ressort de torsion dont les déformations sont lues au 
moyen d'un miroir, et l'aimant est rendu mobile autour d'un axe vertical. Cette méthode a déjà été décrite( $\left.{ }^{\dagger}\right)$. Ses avantages sont la faible étendue dans laquelle les champs magnétiques intenses sont nécessaires et l'extrême simplicité des mesures relatives $\left({ }^{2}\right)$. Nous nous proposons de discuter ici deux sortes d'erreurs auxquelles elle est exposée et qui, tout en pouvant en principe être rendues aussi petites que l'on voudra, la rendent moins apte à déterminer la loi de l'approche vers la saturation qu'à comparer deux états successifs peu différents de la même substance pour la même valeur du champ.

Infuence de la non-uniformité du champ. - L'ellipsoïde est placé au centre d'un champ magnétique de révolution. Ce champ est maximum en ce point pour un déplacement dans le plan équatorial, $y$, et minimum pour un déplacement axial $x$. Il est donné par le développement :

$$
\mathrm{H}=\mathrm{H}_{0}+\frac{x^{2}}{1.2}\left(\frac{\partial^{2} \mathrm{H}}{\partial x^{2}}\right)_{0}+\frac{y^{2}}{1.2}\left(\frac{\partial^{2} \mathrm{H}}{\partial y^{2}}\right)_{0}+\ldots,
$$

qui devient, en remarquant que le potentiel magnétique satisfait à l'équation $\Delta V=0$, et en introduisant les coordonnées polaires $r$ et $\theta$ :

$$
\mathrm{H}=\mathrm{H}_{0}+\frac{r^{2}}{2}\left(\frac{\partial^{2} \mathrm{H}}{\partial x^{2}}\right)_{0}\left(\cos ^{2} \theta-\frac{1}{2} \sin ^{2} \theta\right) .
$$

Or l'énergie d'une particule magnétique de volume $d v$, partie de l'ellipsoïde supposé très allongé, dont l'aimantation, supposée de grandeur constante I, est parallèle au champ, est :

$$
\mathrm{W}=-\mathrm{I} \cdot \mathrm{H} \cdot d v,
$$

et par suite le moment exercé sur elle par le champ:

$$
d \mathbf{M}^{\prime}=-\frac{\partial \mathrm{W}}{\partial \theta}=\frac{3}{2} d v . \mathrm{I}\left(\frac{\partial^{2} \mathrm{H}}{\partial x^{2}}\right)_{0} r^{2} \sin \theta \cos \theta
$$

(l) P. Weiss, J. de Phys., $4^{\circ}$ série, t. V1, p. $663 ; 1907$.

(2) Pour comparer les intensités daimantation $\mathrm{I}$ et $\mathrm{I}^{\prime}$ et les aimantations spécifiques $\sigma$ et $\sigma^{\prime}$ il faut tenir compte de ce que $v$ varie par la dilatation thermique. En appelant $d$ et $d^{\prime}$ les densités on a :

$$
\frac{\mathrm{I}}{\mathrm{I}^{\prime}}=\frac{\sqrt{\mathrm{M}}}{\sqrt{\overline{\mathrm{M}}^{\prime}}} \cdot \frac{\sqrt{d}}{\sqrt{\bar{d}^{\prime}}} \quad \text { et } \quad \frac{\sigma}{\sigma^{\prime}}=\frac{\sqrt{\mathrm{M}}}{\sqrt{\overline{\mathrm{M}^{\prime}}}} \frac{\sqrt{d^{\prime}}}{\sqrt{\bar{l}}} \text {. }
$$

La dilatation aux basses températures n'étant pas connue, nous avons omis la correction provenant de ce que le rapport des densités est différent de l'unité. On peut évaluer cette correction à 0,00 , , en valeur relative. 
AIMANTATION AUX TRÈS BASSES TEMPÉRATURES 567

Le couple exercé par le champ $\mathbf{I I}_{0}$ sur l'ellipsö̈le a pour expression :

$$
\mathbf{M}=\left(\mathbf{N}_{1}-\mathbf{N}_{2}\right) I^{2} v \cdot \sin \varphi \cos \varphi \cdot
$$

Dans les champs très intenses, la condition du parallélisme de l'aimantation au champ extérieur est remplie, malgré les champs démagnétisants de l'ellipsoïde, et l'on a $\theta=\varphi$. Le moment parasite $d \mathbf{M}^{\prime}$ varie donc en fonction de l'azimut de la substance comme le couple principal M. La valeur maxima du couple $d \mathrm{M}^{\prime}$ est :

$$
d \mathbf{M}^{\prime}=\frac{3}{4} d v \cdot \mathrm{I}\left(\frac{\partial^{2} \mathrm{H}}{\partial x^{2}}\right)_{0} r^{2}
$$

qui devient pour tout l'ellipsoïde :

$$
\mathrm{M}^{\prime}=\frac{3}{20} \mathrm{I}\left(\frac{\partial^{2} \mathrm{H}}{\partial x^{2}}\right)_{0} \cdot v \cdot a^{2}
$$

où $a$ est le demi-grand axe de l'ellipsoïde.

Supposons que le champ de l'aimant varie en restant semblable à lui-même et appelons $(\mathbf{l}-\varepsilon) \mathrm{H}_{0}$ le champ à une distance de $\mathbf{1}$ centimètre de l'axe suivant l'axe des $y$. Alors :

$$
\varepsilon \mathrm{H}_{0}=-\frac{1}{2}\left(\frac{\partial^{2} \mathrm{H}}{\partial y^{2}}\right)_{0}=\frac{1}{4}\left(\frac{\partial^{2} \mathrm{H}}{\partial x^{2}}\right)_{0}, \quad \text { d'où } \quad \mathrm{I}^{\prime}=\frac{6}{5} \mathrm{I} \varepsilon \mathrm{H}_{0} \cdot v \cdot a^{2},
$$

et le rapport des couples maxima :

$$
\frac{\mathrm{M}^{\prime}}{\mathrm{M}}=\frac{6}{5} \varepsilon a^{2} \frac{\mathrm{H}_{0}}{\left(\mathrm{~N}_{1}-\mathrm{N}_{2}\right) \mathrm{I}} .
$$

L'avant-dernière équation montre que la valeur absolue du couple parasite croît proportionnellement au champ quand l'aimantation reste constante. Au lieu de trouver pour la saturation une asymptote horizontale, on trouvera une asymptote inclinée. Ce fait a été constaté très nettement dans certaines expériences antérieures. A clıamp égal le couple parasite croît avec I. S'il est inappréciable pour les plus grandes valeurs de I observables avec un appareil, à plus forte raison l'est-il pour les plus petites. La dernière équation montre que l'importance relative du couple de non-uniformité croìt quand l'intensité diminue. Il sera donc particulièrement à craindre dans des recherches où, passant à des intensités d'aimantation plus faibles on augmente la sensibilité en changeant le ressort. 
Pour nos mesures, il suffit de pouvoir se faire une idée de l'ordre de grandeur de l'erreur. A cet effet la non-uniformité du champ a été mesurée pour trois valeurs de $y$ et a été trouvée proportionnelle à $y^{2}$ avec la valeur numérique $\varepsilon=0,0087$. Avec $a=0,1$, il en résulte :

$$
\frac{\mathbf{M}^{\prime}}{\mathrm{M}}=0,00023 \frac{\mathrm{H}_{0}}{\left(\mathrm{~N}_{1}-\mathrm{N}_{2}\right) \mathrm{I}} .
$$

Or, pour les ellipsoïdes employés, $\mathrm{N}_{1}=1,90, \mathrm{~N}_{2}=5,59$, et pour le fer $\left(\mathrm{N}_{1}-\mathrm{N}_{2}\right)$ I est voisin de 6.600 gauss, pour le nickel de 1.800 gauss. La correction dépasserait donc à peine un millième pour le fer, elle atteindrait quelques millièmes pour le nickel.

Réaction de l'ellipsoïde sur les pièces polaires. - Lorsque les sommets de l'ellipsoïde sont dans le voisinage immédiat des surfaces frontales des pôles des aimants, ils ont une influence sensible sur la répartition du magnétisme dans les pièces polaires, et le couple s'en trouve augmenté. Ce fait a été mis en évidence au moyen d'expériences préalables, faites avec un plus grand électro-aimant dont les pièces polaires planes avaient 10 centimètres de diamètre, en mesurant le couple maximum exercé sur un ellipsoïde par le même champ de 9.770 gauss, obtenu avec des distances très différentes des pôles, en donnant au courant des valeurs convenables. On a trouvé ainsi, pour un ellipsoïde de fer de 9 millimètres de longueur et de 4 millimètres de diamètre équatorial :

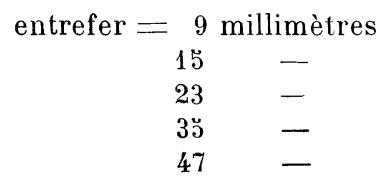

$$
\begin{aligned}
\text { couple maximum } & =335,5 \\
& =320,45 \\
& =319,32 \\
& =319,18 \\
& =319,08
\end{aligned}
$$

La manière dont cette quantité varie montre que la variation n'est pas un effet de la non-uniformité du champ, car c'est précisément quand la perturbation a sa plus grande valeur que, par suite du rapprochement des pièces polaires planes, le champ est le plus uniforme. A partir de 23 millimètres, l'effet est insensible et le couple devient constant.

b) Électro-aimant. -- 11 résulte des renseignements donnés cidessus sur l'influence de la proximité des surfaces polaires et de l'ellipsoïde que l'écartement des pôles doit être environ trois fois la longueur de l'ellipsoïde. L'épaisseur totale des quatre parois du tube Dewar et du tube ajusteur $(\S 2 c)$ ne peut guère descendre au- 

RECHERCHES SUR L'AIMANTATION AUX TRĖS BASSES TEMPÉRATURES.
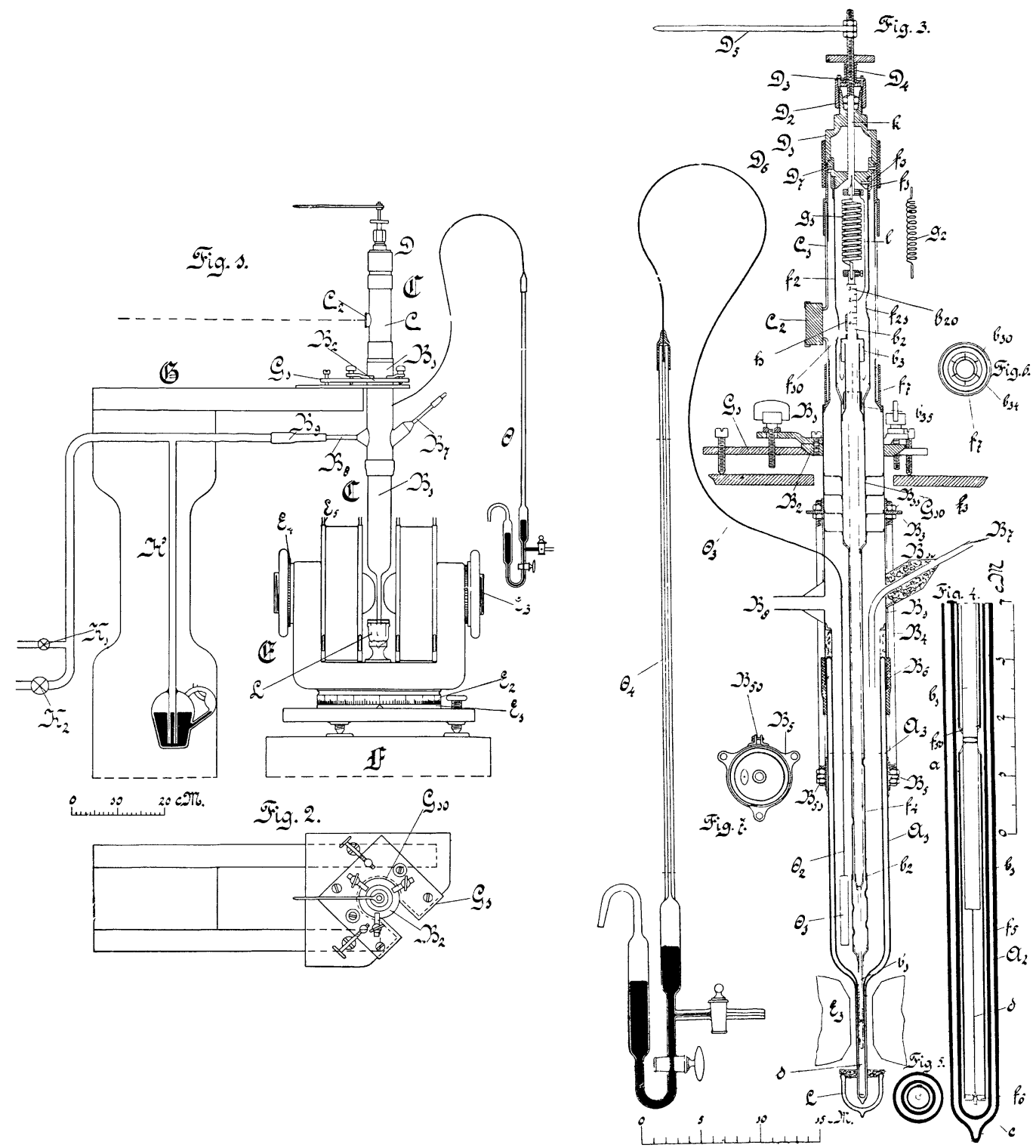
MIMANTATION AUX TRĖS BASSES TEMPÉRATURES 569 dessous de 5 millimètres. En tenant compte à la fois de la dilficulté d'obtenir des champs intenses dans des espaces étendus et de celles de la réalisation exacte d'ellipsoïdes très petits, nous nous sommes arrètés à un entrefer de 9 millimètres et à des ellipsoïdes de 3 millimètres de longueur.

On peut obtenir dans cet entrefer des champs relativement intenses (jusqu'à 25.000 gauss), avec un aimant dont les noyaux ont 9 centimètres de diamètre. L'électro-aimant a servi précédemment à des expériences magnétiques aux températures élevées. Il a déjà été décrit(1) et est représenté schématiquement dans la fig. 1. Relativement léger (132 kilogrammes) et facılement transportable pour sa puissance, il a pu être étudié à Zurich et employé à Leyde. Il possède un mouvement micrométrique des pièces polaires que l'on peut avancer et reculer au moyen de manettes-écrou dont on repère la position sur des cercles divisés. Il est mobile autour d'un axe vertical, grâce à un support comportant une couronne de billes se mouvant dans des gorges annulaires. Les azimuts sont lus au moyen d'un trait de repère fixe, sur une graduation cylindrique portée par la partie mobile du support.

Chacune des bobines reçoit 1.5000 tours de fil de $2^{\mathrm{mm}}, \breve{s}$ et a une résistance de $2 \mathrm{ohm}$ environ. Comme elle est destinée à un courant de 10 ampères en régime normal, et que celui-ci peut atteindre 2ơ ampères pendant un temps relativement court, on dispose d'un nombre d'ampères-tours pouvant aller jusqu'à 7ö.000. La circulation d'eau dans les doubles parois des bobines a pour effet de perriettre une durée à peu près double des expériences, et surtout de protéger les pièces polaires et les noyaux contre l'échauffement. Cet échauffement a de·nombreux inconvénients dont le moindre n'est pas d'altérer d'une manière appréciable la valeur du champ. La dilatation thermique des noyaux relativement longs peut, en effet, faire varier d'une manière appréciable un entrefer relativement court.

c) Appareil cryogène. - La nécessité de monter l'équipage mobile, portant l'ellipsoïde dans un espace fermé recevant l'hydrogène liquide et l'atmosphère gazeuse exige un appareil cryogène assez compliqué représenté dans la vue d'ensemble $(f g$. 1) et en coupe $(f g .3)$. Il se compose essentiellement de trois parties coaxiales de forme tubulaire qui sont, en allant de l'extérieur à l'intérieur, le

(1) G. Zinnel, Revue électrique, 20 juin 1909, et Elelitrotech. Zeitschr., t. XXX, p. $446 ; 1909$.

J. de Phys., $4^{\circ}$ série, t. IX. (Juillet 1910.) 
tube enveloppe, le tube ajusteur $f$ et l'équipage b. Le tube-enveloppe se compose, de bas en haut, du tube Dewar A, du tube de laiton B, du tube de verre C, et de la chape D qui ferme l'appareil.

Equipage. - L'ellipsoïde $a$ (fig. 3 et 4) peut tourner autour d'un axe vertical avec l'équipage $b$ dans lequel il est fixé. Cet équipage. est composé sur la plus grande partie de sa longueur d'un tube en argentan $(b)$, matière peu magnétique, rigide et mauvaise conductrice de la chaleur, et à sa partie inférieure de la tige de cuivre $b$, dont le magnétisme propre est très faible. ll est suspendu à la tige $k$ par le ressort hélicoïdal $g_{1}\left(g_{1}\right.$ a servi pour le fer et le cobalt, le ressort plus faible qui a servi pour le nickel et la magnétite est représenté à côté en $g_{2}$ ). Pour assurer la stabilité de l'équilibre et empêcher l'ellipsoïde d'aller se coller contre les pôles de l'aimant, l'équipage est fixé en bas par un fil de platine iridié de $0^{\mathrm{mm}}, \mathbf{1}$ de diamètre et dont la torsion n'intervient que comme une correction $(4)$. Le tube $b$ et la tige $b_{1}$ sont soigneusement dressés au tour. l'ellipsoïde $a(f i g$. 4) y est fixé dans un trou de diamètre égal à celui de l'équateur et de l'ellipsoïde. Lorsqu'il s'agit de la magnétite ou du nickel, on peut le fixer avec un peu de cire; dans le cas du f $\bullet$ et du cobalt, les efforts étaient suffisamment grands pour qu'il soit nécessaile de le sertir en l'enfonçant à force dans la cavité après l'avoir enveloppé d'une très mince lame de cuivre. La tige $b_{1}$ et le tube très mince $b$, en argentan, transmettent (fig. 3 ) la rotation. de l'ellipsoïde (1) au miroir $h$ qui, par l'ouverture $f_{10}$ et la fenêtre $\mathrm{C}_{2}$ ( $f g .1$ et 3 ), permet la lecture de la torsion du ressort $g_{1}$ sur une échelle de verre de $1^{\mathrm{m}}, \breve{0} 0$ de longueur, divisée en demi-millimètres, éclairée par des miroirs sphériques $\left(^{2}\right)$, et placée à $4^{\mathrm{m}}, 325$ de distance. La tension du ressort est.réglée en faisant monter ou descendre la tige $k$ (fig. 3) qui pénètre dans la chape par le presseétoupe $D_{2}$. Cette opération s'effectue en faisant tourner l'écrou $D_{4}$ pendant que $D_{\mathfrak{y}}$ est maintenu immobile. La tension est lue, par l'ouverture $f$, sur l'échelle $b$ à l'aide du pointeur $l$. Par une opération préliminaire sur l'appareil démonté, dans laquelle on suspend à l'équipage un poids connu, on détermine la division de l'échelle $b$

(1) Une petite torsion de ce tube n'intervient pas. La partie de l'appareil qui joue effectivement le rôle de ressort est comprise entre le miroir et la chape. La torsion de la partie de l'appareil au-dessous du miroir transmet simplement le couple, son seul effet est de changer imperceptiblement l'azimut de l'aimant.

$\left({ }^{2}\right)$ H. Kamerlixgh Oxnes, Communic. Physical Labor., n².3 ; Leiden. 1896. 
AIMANTATION AUX TRES BASSES TEMPÉRATURES 571

qui correspond à la tension pour laquelle on se propose de régler l'appareil. Les vibrations que cet appareil tend à prendre comme une corde tendue, sous l'influence des trépidations, sont amorties par l'amortisseur $b_{3}$ à ailettes ( $f g .3$ et 6 ) plongeant dans un bain d'huile annulaire, divisé en compartiments par des parois $b_{3}$, fixées sur un cylindre qui tourne à frottement doux dans le tube porteur et peut ainsi être entraîné par les ailettes $b_{30}$, quand l'équipage vient à effectuer une rotation un peu grande $\left({ }^{1}\right)(\$ \mathbf{4})$.

Les ailettes doivent plonger entièrement dans l'huile pour éviter des couples provenant de la capillarité $(\$ 4)$ qui pourraient ne pas être négligeables. Dans les champs forts, les courants de Foucault amortissent très bien les vibrations de torsion.

Tout l'équipage avec le ressort est suspendu dans le tube ajusteur $f$, dont la partie supérieure est vissée à la chape D (fig. 3 et 1), laquelle porte aussi la tige $k$ et repose sur le tube de verre C. Le tuhe ajusteur, composé des parties $f_{2}, f_{3}, f_{7} \cdot f_{5}$, diminue de diamètre à trois reprises, la partie inférieure $f_{3}$ entourant d'aussi près que possible la tige $b_{1}$ de l'équipage. Contre le fond $f_{t ;}(f i g$. 4) bute le cône $\mathrm{C}$ soudé au fil tenseur $d$ et le relenant. Ce cône peut entrer dans le fond $f_{6}$ par une coupure (fig. 3 ). Quand l'appareil est monté, le tube ajusteur plonge dans le tubé Dewar A. Le tube porteur comme le tube $b_{2}$ formant l'équipage mobile est en argentan.

Pour monter dans le tube enveloppe, le tube ajusteur, avec l'équipage qu'il contient, on le visse à la chape D. Puis on place cette chape sur la garniture en bronze mastiquée en haut du tube de verre C, on serre les vis $\mathrm{C}_{7}$ et on rend le joint hermétique au moyen du tube de caoutchouc collé $\mathrm{D}_{6}$. Le bas du tube de verre $\mathrm{C}$ est mastiqué dans une deuxième garniture en bronze soudée au tube de laiton B. Ce tube porte en son milieu un anneau $B_{3}$ sur lequel reposent les écrous $B_{31}$ des tringles $B_{4}$ portant le tube Dewar. Celui-ci, dont la partie étroite $\mathrm{A}_{2}$ et la moitié inférieure de la partie plus large jusqu'au niveau $\mathrm{A}_{3}$ sont argentées (la partie supérieure esi restée transparente pour que l'on puisse s'assurer que l'on ne verse pas trop d'hydrogène liquide), glisse dans le tube $B_{4}$ en mallechort et bute contre un anneau en bois contre lequel il est pressé par les tringles $\mathrm{B}_{\mathfrak{1}}$. La fig. 7 montre comment l'anneau $\mathrm{B}_{\mathfrak{3}}$ en laiton mince, auquel

(1) Il importe que l'huile soit privée d'air et qu'il ne se trouve pas de bulles sous l'huile, parce que l'appareil doit être évacué une fois entièrement monté. 
aboutissent on bas les tringles $B_{1}$, est fixé sur le tube Dewar, protégé par une couche de papier, au moyen d'une bride serrée par la vis $B_{3 \varkappa}$.

La partie inférieure du tube Dewar a un diamètre extérieur de 8 millimètres et un diamètre intérieur de 5 millimètres. Les parois de verre ont unc épaisseur de 0,5 millimètre, de sorte qu'il ne reste pour la distance des deux faces argentées en regard que $0, \check{\text { milli- }}$ mètre.

Pour centrer l'appareil, on le fait reposer, par l'anneau $B_{2}$ de l'enveloppe, sur un support auxiliaire. Le tube Dewar étant enlevé on passe la partie étroite $f_{\mathfrak{z}}$ du tube ajusteur dans un calibre approprié que lon fixe. On peut ensuite retirer de ce calibre un anneau, le remplacer par un autre etainsi en augmenter le diamètre de manière qu'il corresponde au diamètre extérieur de la partie étroite du tube Dewar $A_{2}$. On met celui-ci exactement en place au mojen des écrous $B_{31}$. Le joint est rendu hermétique au moyen du tube de caoutchouc $B_{6}$ collé et ligaturé avec des fils de cuivre. Le mode de fixation du tube Dewar avec les tringles $\mathrm{B}_{4}$ résiste à la déformation par évacuation, après laquelle le centrage ne demande que de très légères retouches.

Dans le tube B est soudé le tube capillaire en acier $\theta_{3}(f g .1$ et 3 ) d'un thermomètre à hélium ( $\left.{ }^{4}\right)$ à réservoir $\theta_{1}(f i g .3$ et 7$)$ en argentan et tige $\theta_{4}$ en verre, qui est monté solidairëment avec le tube enveloppe. La quantité d'hélium est mesurée de telle façon, que pour le point d'ébullition de l'oxygène, le mercure se fixe au bas de la tige et pour le point de solidification de l'hydrogène, il atteigne la partie supérieure. Lorsque, comme c'est le cas avec l'hydrogène bouillant sous la pression normale, la température est assez bien connue sans la lecture du thermomètre, celui-ci est encore nécessaire comme indicateur du niveau du gaz liquéfié qui devient invisible au-dessous de $A_{3}$. Aussitôt que le niveau descend au-dessous de la partie supérieure du réservoir $\theta_{1}$, le mercure baisse dans la tige $\theta_{4}$.

d) On procède au réglage de l'aimant indépendamment de celui du tube ajusteur et de l'équipage par rapport au tube Dewar. Son axe de rotation est rendu vertical et l'entrefer est centré sur cet axe, puis l'anneau à évidement sphérique $\mathrm{G}_{10}(f i g .1,2$ et 3 ) est amené sur l'axe de l'aimant. Cet anneau est porté par une plaque de laiton,

(1) Comp. L'appareil pour la liquéfaction de l'hélium, H. Kamerlingh Onnes, Communic. Physical Labor., $\mathbf{n}^{\circ} 108$; Leiden, 1908. 
AIMANTATION AUX TRÈS BASSES TEMPÉRATURES 573

qui elle-même est fixée par l'intermédiaire de deux madriers de sapin au pilier en pierre de taille II.

On transporte ensuite l'appareil cryogène du support provisoire à sa place définitive en faisant reposer la surface sphérique de l'anneau $B_{2}$ dans l'évidement de l'anneau $G_{10}$ dans lequel elle s'adapte exactement, et on achève le centrage de la partie étroite du tube Dewar sur l'axe de l'aimant au moyen des vis à ailettes de l'anneau $\mathrm{B}_{2}$. Ce centrage doit être exact, car l'aimant doit pouvoir tourner librement, et l'espace entre le tube Dewar et les pôles n'est qu'un demi-millimètre de chaque côté. Il réussit d'ailleurs facilement à $0^{\mathrm{mux}}, 2$ ğ près.

e) L'introduction de l'hydrogène liquide se fait par le tube $\mathrm{B}_{7}$ en argentan (cf. Comm. n⿳9 94 f), le gaz qui s'évapore s'échappe par $\mathrm{B}_{8}\left(f g .3\right.$ et 1 ) et les robinets $\mathrm{K}_{1}, \mathrm{~K}_{2}$, dont l'un conduit à un gazomètre et l'autre est en communication avec une pompe à vide. En manipulant les robinets, on règle la tension de vapeur qui est lue au

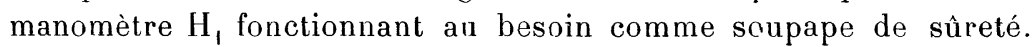
Dans les expériences au voisinage du point de solidification de l'hydrogène, on prend soin d'opérerà une pression un peu supérieure à celle du triple-point.

Avant d'introduire l'hydrogène liquide par $B_{7}$ qui est fermé par un tube de caoutchouc et un bouchon de verre, on fait le vide dans l'appareil par $K_{2}$. L'étanchéité parfaite est de rigueur, parce que des traces d'air se solidifieraient dans l'hydrogène liquide et s'accumuleraient, par suite de l'attraction magnétique, dans le voisinage de l'ellipsoïde.

Pour empêcher que l'hydrogène en ébullition ne refroidisse à la partie supérieure de l'appareil là où se trouve le ressort, le tube $f_{4}$ (fig. 3) a été percé d'un certain nombre de grands trous n'affectant pas sa rigidité torsionnelle; en outre il glisse à frottement doux entre les écrans de cuivre qui entourent $f_{3}$ et sont soudés à B. Pour éviter l'ébullition explosive( ('), on met un peu d'ouate au fond du tube Dewar et des mèches d'ouate autour du tube ajusteur. Le renouvellement de l'hydrogène liquide se fait de la même manière que le remplissage. Un seul remplissage suftit généralement pour plusieurs séries de mesures. La partie inférieure du tuke Dewar, dont l'extrémité n'avait pu être argentée, est protégée par un petit vase L

(1) Lorsqu'elle se produit, il faut s'assurer que des gouttelettes projetées n'ont pas refroidi l'huile de l'amortisseur. 
contenant de l'air liquide. Lorsque, l'appareil une fois rempli, la partie au-dessus des écrans est revenue à la température ordinaire, on ne s'aperçoit presque plus qu'il y a de l'hydrogène dans l'appareil, à moins que le niveau du liquide ne soit au-dessus de $A_{3}$.

A la longue il se condense un peu d'humidité sur le tube Dewar. Pour éviter qu'elle coule entre les pièces polaires, on entoure le tube de papier buvard en $A_{1}$. Un courant d'air est lancé sur le tube entre les pôles. Les surfaces polaires ne sont donc aucunement affectées par les općrations cryogéniques.

f) Les ressorts sont en bronze phosphoreux. Cette matière n'est pas magnétique et n'a que de faibles déformations résiduelles. On peut réaliser un ressort de constante donnée en enroulant en hélice soit un fil fin et court, soit un fil plus gros et notablement plus long. De ces deux ressorts celui qui contient le plus de matière travaille avec des déformations spécifiques moindres et par conséquent une élasticité plus parfaite. Il a été tenu compte de cette circonstance. Les ressorts sont munis dans l'axe d'allonges rigides et sont fixés avec des vis dans l'équipage et dars la tige $k(f g .3)$. Les spires ne se touchent pas. La température du ressort est déterminée par un thermomètre à mercure appliqué contre la chape $\mathrm{D}$ et enveloppé de laine avec celle-ci. Les constantes des deux ressorts employés sont 261000 et 22300 dynes-centimètres par unité d'arc. Pour la correction de l'influence du fil tenceur et des variations de température du ressort, voir au $\$ 4$.

Les ellipsoüdes de fer, de nickel et de cobalt ont 3 millimètres de longueur et $1^{\mathrm{mm}}, 333$ de diamètre équatorial. Ils ont été exécutés avec une grande précision par la Suciété genevoise pour la construction d'instruments de mécanique et de physique. Le travail a été fait au tour sous un microscope grossissant 30 fois et muni d'une chambre claire permettant de superposer l'objet à un dessin à grande échelle. Quelques mesures laites à la machine à diviser ont moritré que les ellipsoïdes sont très exacts.

Le ler a été obtenu en londant, au four électrique à résistance, du fer électrolytique pur de Merck dans une atmosphère d'azote et dans une nacelle de magnésie. Le nickel et le cobalt ont été obtenus de la même nanière en partant de nickel et de cobalt pulvérulents, extrèmement purs, préparés spécialement par Merck pour ces recherches. La magnétite a été olılenue en taillant un ellipsoïde approché dans une goutte de magnetite fondue très pure, obtenae en 
AIMANTAION AUX TRÈS BASSES TEMPERATURES 575 chanffant le sesquioxyde pur de Merck avec le chalumean oxhydrique. L'expérience ayant montré que l'on n'obtient réellement de la magnétite (i) qu'en calcinant à très haute température pour faire partir les derniers restes d'oxygène en excès, cette opération a été faite dans une coupelle en iridium. Il a été préparé de même des ellipsoïdes grossièrement approchés dans du chrome et du manganèse de Goldschmidt et du vanadium de Moissan. Il est facile de se rendre compte qu'il n'est pas nécessaire que les ellipsoïdes soient réalisés avec une grande perfection pour des mesures relatives; cela a d'ailleurs été constaté expérimentalement pour la magnétite, pour laquelle on a trouvé avec différents échantillons grossièrement taillés en ellipsoïdes de formes diverses des courbes de variation thermique aux hautes températures coïncidant avec la courbe théorique et par conséquent entre elles.

\section{§ 3. - MARChE des eXPÉriences.}

Comme cela a été dit dans l'introduction, nous nous sommes proposé non de faire des mesures absolues, mais de comparer les intensités d'aimantation dans les champs élevés à la température ordinaire et à celle de l'hỵdrogène liquide, les variations attendues étant une fraction assez petite de la quantité à mesurer. Il semblait donc indiqué de croiser les mesures à la température ordinaire et à celle de l'hydrogène liquide pour une même valeur du champ. Mais le passage d'une température à l'autre exige des opérations d'une trop grande durée et une attente trop longue pour que l'on puisse procéder ainsi. On a donc commencé, en général, par des mesures à température ordinaire en donnant au champ toute une série de valeurs. Puis on a fait une série analogue à basse température, et, la température ordinaire rétablie, on a repris quelques points pour constater que l’appareil n'avait pas subi d’altération.

Une série de mesures se décompose elle-même en deux parties. On commence par déterminer par tàtonnements méthodiques, pour toutes les valeurs des champs, les azimuts de l’aimant pour lesquels le couple est maximum. On trouve ainsi deux azimuts symétriques par rapport au grand axe de l'ellipsoïde, qui donnent des couples de

(1) Voir aussi P. Weiss, Arch. cles Sc. phys. et nat., 4 pér., t. XXIX, p. 175: 1910; et $J$. de Phys., $4^{\ominus}$ série, t. 1X, p. $373 ; 1910$. 
signes contraires. Cette détermination se fait avec une précision de $\mathbf{1}^{\circ}$ à $0^{\circ}, \breve{5}$, qui est largement suffisante. Ensuite, la mesure proprement dite consiste, après avoir amené l'aimant sans courant dans l'un des deux azimuts, à fermer le courant et à observer l'élongation immédiatement après. On coupe aussitôt le courant, on oriente l'aimant dans l'azimut sỵmétrique, on rétablit le courant et on observe encore l'élongation. Ces opérations étant de faible durée, la déformation visqueuse du ressort n'intervient pas. La distance entre les deux points lus sur l'échelle donne deux fois le couple à mesurer, indépendamment de l'influence de l'aimantation résiduelle qui reste après la rupture du courant et dont le couple était d'ailleurs extrêment faible. Le champ était connu en fonction du courant d'excitation de l'ampèremètre. C'est le même ampèremètre (Siemens et Halske, sans coefficient de température) qui a servi dans l'étude préalable de l'aimant et les mesures définitives. Ce mode de repérage du champ était largement suffisant pour le but poursuivi. La position des pôles a été repérée par la lecture des tambours divisés de l'aimant et contrôlée au moyen d'un calibre exactement ajusté à la longueur de l'entrefer. Les champs donnés plus loin sont corrigés des champs démagnétisants des ellipsoïdes.

\section{§4. - Corrections et controles, déterminations auxiliaires.}

Le magnétisme propre de l'équipage n'est pas assez faible pour que sa correction puisse être négligée. On a donc fait une série de mesures à blanc, sans substance, à la température ordinaire et à celle de l'hydrogène liquide. On a obtenu ainsi avec le ressort le plus faible:

Tableau I. - Correction du magnétisme du support.

\begin{tabular}{|c|c|c|c|c|}
\hline \multicolumn{2}{|c|}{4000 gauss } & \multicolumn{2}{|c|}{$\begin{array}{c}\text { Temp. ord. } \\
0^{\mathrm{cm}}, \mathbf{1 8}\end{array}$} & $\begin{array}{c}t=20^{\circ}, 3 \mathrm{~K} \\
0,26\end{array}$ \\
\hline 8000 & - & & ,29 & 0,48 \\
\hline 12000 & - & 0 & ,36 & 0,61 \\
\hline 16000 & - & 0 &, 43 & 0,73 \\
\hline 20000 & - & 0 & , 50 & 0,86 \\
\hline 24000 & - & 0 &, 57 & 0,98 \\
\hline
\end{tabular}

Pour le ressort plus fort, ces corrections sont réduites dansle rapport de $\frac{22300}{261000}$, elles sont donc très petites. Des mesures directes 
AIMANTATION AUX TRÈS BASSES TEMPÉRATURES 577

ont montré que les valeurs ainsi calculées étaient exactes. Cela revient à dire que le magnétisme propre du support n'avait pas été altéré par les divers démontages.

Une correction à apporter au rapport des couples est celle de la variation de rigidité du fil tenseur au bas de l'équipage à la température ordinaire et à celle de l'hydrogène liquide. A cet effet on a déterminé le rapport de la constante de torsion du fil de platine iridié, à la température ordinaire et à celle de l'hydrogène liquide, à celle du plus faible des deux ressorts en bronze phosphoreux, à la température ordinaire. On s'est servi d'un appareil identique à celui qui a été décrit, mais dont la chape pouvait tourner d'un angle connu par rapport au tube-enveloppe. La position de la chape était repérée au moyen d'un miroir et d'une échelle placée à $17 \mathrm{ă}^{\mathrm{cm}}, 9$. On donnait à la chape une rotation mesurée, voisine de $360^{\circ}$. Cette rotalion était la somme des torsions du ressort et du fil pour un même couple. Une lecture au miroir porlé par l'équipage donnait la torsion du fil. On a trouvé ainsi pour le rapport de la constante du fil à celle du ressort :

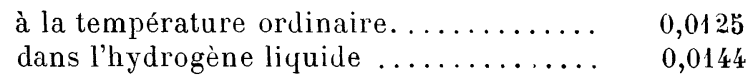

déterminations dans lesquelles le chiffre des dix-millièmes estincertain. La correction atteint donc deux millièmes pour le ressort faible et deux dix-millièmes pour le ressort fort.

Le coefficient de température du ressort en bronze phosphoreux a été déterminé en mesurant la période d'un même système oscillant, le ressort étant à la température ordinaire et dans la vapeur d'eau bouillante. On a trouvé ainsi le coefficient de température :

$$
\mathrm{K}=-0,000 \mathrm{3} 3
$$

au moyen duquel les observations ont été ramenées à la même température du ressort.

La constance de la température du bain liquide dans toute l'étendue du tube Dewar a été constatée à moins de $0^{\circ}, 1$ près en déterminant dans un tube identique la température à diverses hauteurs avec un thermomètre à résistance en platine qui, placé à côté du thermomètre $\theta$, donnait précisément les températures correspondant aux tensions de vapeur. 
Phénoménes cupillaires de l'huile de l'amorlisseur. - On a pris soin de remplir suffisamment le godet de l'amortisseur pour que la surface de l'huile soit traversée par la bague cylindrique qui porte les ailettes sur laquelle la tension superficielle ne peut exercer aucun couple. Mais on a voulu, néanmoins, se rendre compte de l'ordre de grandeur des forces entrant en jeu dans les phénomènes capillaires en les augmentant énormément. A cet effet, dans un amortisseur aussi semblable que possible à celui en usage, on a mis une quantité d'huile telle que la surface soit traversée par les ailettes fixes et mobiles. La partie mobile a été suspendue à un fil de platine iridié de 20 centimètres de longueur et de $0^{\mathrm{mm}}, 1$ de diamètre, et ses déviations-étaient lues, au moyen d'un miroir, sur une échelle à 2 mètres. On a placé successivement le récipient d'huile dans deux azimuts où le rapprochement des ailettes devait produire des couples de signe contraire. On a observé une déviation de 5 centimètres sur l'échelle. Le couple est donc de l'ordre de deux millièmes de celui qui est exercé sur l'ellipsoïde de nickel.

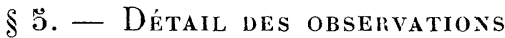

Nickel. -- 11 a d'abord été fait sur le nickel une première série d'observations à la température de $17^{\circ}, 2 \mathrm{C}$.

Tableau II.

$\begin{array}{rccc}\text { H gauss. } & \text { I2 cm. de l'échelle } & \text { H gauss. } & \text { I2 cm. de l'ćchelle } \\ 2230 & 89,42 & 17760 & 90,50 \\ 6230 & 89,97 & 20300 & 90,66 \\ 10270 & 90,12 & 21540 & 90,79 \\ 13280 & 90,34 & 22760 & 90,81\end{array}$

Toutes les lectures sont corrigées du rapport de la tangente de la double déviation à la double déviation.

Le zéro, déterminé par la moyenne des observations à droite et à gauche, est resté invariable à quelques dixièmes de millimètre près. Après cette série, l'appareil a été détérioré accidentellement, démonté, remonté, ce qui a changé quelque peu la grandeur des déviations. La variation avec $H$ étant établie par la série ci-dessus, on a pris seulement deux points à la température ordinaire, avant et après l'hydrogène liquide. 
T.BLEAU III.

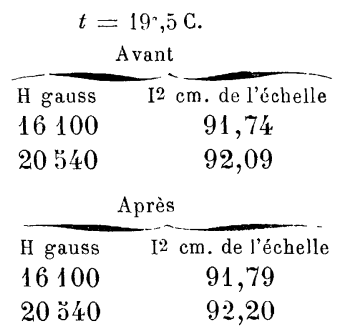

\begin{tabular}{|c|c|}
\hline H gauss & $I^{2} \mathrm{~cm}$. de l'échelle \\
\hline 1780 & $93, \because \% 7$ \\
\hline 5410 & 100,49 \\
\hline 8050 & 101,34 \\
\hline 11830 & 101,84 \\
\hline 16100 & 102,13 \\
\hline $190 \breve{0}$ & 102,34 \\
\hline $20 丂^{\prime} \mathrm{H}^{\circ}$ & 102,31 \\
\hline 22020 & 102.48 \\
\hline 22840 & 102,49 \\
\hline
\end{tabular}

Dans cette série, le zéro, déterminé par la moyenne des observations à droile et à gauche, a varié de 2 millimètres environ.

$$
\begin{aligned}
& \text { Pour } \mathrm{H}=16100 \text { gauss } \quad \frac{\mathrm{I}_{20,3 \mathrm{~K}}}{\mathrm{I}_{19 \circ \mathrm{iC}}}=1,0 \% 49 \\
& \mathrm{H}=20540 \quad=\frac{1,0547}{1,0548}
\end{aligned}
$$

non corrigée de la dilatation thermique.

Cobalt. - Les mesures sur le cobalt n'ont pas abouti au résultat cherché. C'est la difficulté extrême de l'aimanter à saturation qui était conmue par les recherches préliminaires qui adéterminé le choix d'un appareil de si petites dimensions. Pour les autres corps on aurait pu se contenter de champs moins intenses et disposer d'un entrefer plus étendu. Dès les mesures à la température ordinaire il s'est manifesté un phénomène imprévu. En prenant la moyenne des lectures à droite et à gauche qui aurait dû donner le zéro de l'appa$\mathbf{r}$ eil, on a obtenu un point variable en fonction du champ. Cette variabilité a été tronvée ensuite environ deux fois plus grande à basse température. Voici quelques nombres qui la mettent en évidence :

(Dans les expériences sur le cobalt, nous donnons le champ extérieur non corrigé du champ démagnétisant de l'ellipsoïde. Ce dernier est, quand la saturation est atteinte, environ 5000 gauss.) 
Tableau IV. - Cobalt 1, température ordinaire.

\begin{tabular}{rcc|c} 
He gauss & I2 cm. de l'échelle & Zéro calc. & Zéro obs. \\
4025 & 17,16 & 76,73 & \\
8050 & 38,14 & 77,47 & \\
12075 & 50,48 & 78,96 & Le zéro vrai \\
19560 & 53,24 & 78,37 & n'a pas été \\
23340 & 53,29 & 78,18 & noté \\
25650 & 53,30 & 78,63 &
\end{tabular}

Cobalt 1 , H solidification $\left(14^{\circ}, 0 \mathrm{~K}.\right)$.

$\begin{array}{rll}4025 & 13,5 & 77,62 \\ 8050 & 32,59 & 78,84 \\ 15820 & 53,23 & 81,93 \\ 19560 & 54,33 & 81,40 \\ 21800 & 54,43 & 81,16 \\ 23340 & 54,45 & 81,02 \\ 24760 & 54,46 & 80,08\end{array}$

78,26

Il y a donc, à côté du phénomène principal, des couples parasites dissymétriques. Il est probable qu'il s'agit de phénomènes magnétocristallins provenant de cristaux élémentaires du cobalt, insuffisamment nombreux dans le petit ellipsoïde pour produire l'isotropie par compensation. Ces phénomènes ont un signe et une grandeur indépendants de celui du phénomène principal, et pourraient même avoir un effet contraire pour les deux azimuts de l'aimant. Ils peuvent devenir très importants si la substance a un plan magnétique plus ou moins caractérisé, et l'on sait par l'exemple de la pyrrhotine qu'ils augmentent quand la température s'abaisse. D'ailleurs la loi d'approche vers la saturation, différente pour le cobalt de ce qu'elle est pour les autres corps, s'accorde bien avec des phénomènes magnétocristallins intenses $\left({ }^{1}\right)$.

Ces expériences ont été répétées sur un deuxième ellipsoïde de cobalt. Les mêmes dissymétries, un peu moins fortes, se sont manifestées. Mais il s'est ajouté un autre caractère qui montre combien les phénomènes parasites peuvent altérer la mesure de la variation de l'intensité d'aimantation : l'aimantation à basse température a été trouvée en apparence plus petite qu'à la température ordinaire. Le tableau suivant contient un extrait des observations faites sur ce deuxième ellipsoïde :

(1) P. Werss, loc. cil. 
Tableau V. - Cobalt $2, \dot{a} t=18^{\circ}, 5 \mathrm{C}$.

$\begin{array}{rccc}\text { He gauss } & \text { I² cm. de l'échelle } & \text { Zéro calc. } & \text { Zéro obs. } \\ 4025 & 20,33 & 77,56 & 77,70 \\ 12075 & 54,16 & 76,49 & \\ 23340 & 59,76 & 76,86 & \\ 25650 & 59,94 & 76,90 & \end{array}$

Cobalt 2, dans $\mathrm{H}$, pression atmosphérique $\left(20^{\circ}, 3 \mathrm{~K}.\right)$.

$\begin{array}{llll}15080 & 53,53 & 76,61 & \\ 23340 & 58,09 & 77,07 & 78,90 \\ 25650 & 58,46 & 77,21 & \end{array}$

Le même ellipsoïde a été démonté et remonté en le noyant dans du ciment Khotinsky, pour éviter un effet supposé de magnéto-striction extraordinairement intense qui aurait été provoqué jusqu'à présent par le forçage de l'ellipsoïde dans la monture. On a trouvé les mêmes phénomènes, mais l'ellipsoïde ayant pu être placé, dans le nouveau montage, sous un autre angle par rotation autour de son axe, le signe du déplacement de zéro en fonction du champ est changé.

Tableau VI. - Cobalt $2, t=16^{\circ}, 5 \mathrm{C}$.

$\begin{array}{cccc}\mathrm{H}_{e} \text { gauss } & \text { I2 cm. de l'échelle } & \text { Zéro calc. } & \text { Zéro obs. } \\ 8050 & 40,99 & 79,12 & 79,45 \\ 19560 & 56,48 & 78,80 & \\ 23340 & 57,07 & 78,89 & \\ 23650 & 57,3 \pm & 78,90 & \end{array}$

Cobalt 2 , H pression atmosphérique, $t=20^{\circ}, 3 . \mathrm{K}$.

$\begin{array}{rrrr}8050 & 34,07 & 78,88 & 70,20 \\ 19560 & 53,21 & 78,24 & \\ 23340 & 54,26 & 78,33 & \\ 25650 & 54,63 & 78,42 & \end{array}$

La seule conclusion qu'il semble permis de tirer de ces expériences sur le cobalt est que l'accroissement del'aimantation du cobalt entre la température ordinaire et celle de l'hydrogène liquide est certainement beaucoup plus petite que celle du nickel ou de la magnétite, autrement elle n'aurait pu être masquée par le phénomène parasite. 


\section{Tableau VII. - Fer.}

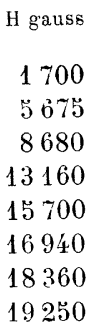

$$
\begin{gathered}
\iota=20^{\circ} \mathrm{C} . \\
95,23 \\
98,47 \\
98,63 \\
98,91 \\
99,04 \\
99,08 \\
99,06 \\
99,07
\end{gathered}
$$

\begin{tabular}{cc}
\multicolumn{2}{c}{$\mathrm{I} \mathrm{cm}$. de l'échelle } \\
\hline$t=20^{\circ}, 3 \mathrm{~K}$. & $t=14^{\circ}, 0 \mathrm{~K}$. \\
H pression atmosphérique & H solidification \\
101,98 & 101,95 \\
102,85 & \\
103,01 & \\
103,31 & \\
103,31 & 103,23 \\
103,27 & \\
103,27 & \\
103,25 & 103,25
\end{tabular}

$$
\begin{array}{rr}
\text { Pour } \mathrm{H}= & 19250 \quad \frac{\mathrm{I}_{200,3 \mathrm{~K}}}{\mathrm{I}_{200 \mathrm{C} .}}= \\
18360 & 1,0209 \\
16940 & 1,0210 \\
15700 & 1,0209 \\
\text { Moyenne........ } & \frac{1,0213}{1,0210}
\end{array}
$$

non corrigée de la dilatation thermique.

Dans toutes les expériences sur le fer, le zéro calculé par la moyenne des lectures reste remarquablement constant. Il ne se déplace en général que de quelques dixièmes de millimètres dans une série, et d'une série à l'autre de 6 millimètres. Les quelques observations à la température de la solidification de $\mathrm{H}$ montrent qu'entre $20^{\circ}, 3 \mathrm{~K}$. et $14^{\circ}, 0 \mathrm{~K}$., il ne se passe rien d'imprévu.

Magnétite. - Nous avons dit que la préparation de la magnétite par calcination du sesquioxyde pur exige une température très élevée pour assurer le départ des derniers restes d'oxygène en excès. Un premier ellipsoïde, provenant d'oxyde de fer incomplètement calciné, n'a donné qu'une intensité d'aimantation un peu supérieure à la moitié de celle qui était attendue, avec une hystérèse très marquée et environ trois fois plus intense à la température de l'hydrogène liquide qu'à la température ordinaire, tandis que, dans toutes les expériences sur les autres substances les effets de l'hystérèse ont été insensibles. De plus, pour cette substance, l'intensité d'aimantation était à peu près la même à la température ordinaire et dans l'hydrogène liquide, passant par un maximum dans l'intervalle.

Ces particularités ont disparu dans un deuxième ellipsoïde taillé dans de la magnétite bien calcinée, mais il s'en est présenté d'autres qui sont inexpliqués jusqu'à présent, mais semblent n'avoir qu'une 
AIMANTATION AUX TRÈS BASSES TEMPÉRATURES 583 importance subordonnée. La position du zéro, calculée comme moyenne des deux lectures sur l'échelle, diffère d'une manière appréciable du zéro observé, tout en restant sensiblement constante dans une série d'expériences à la même température. Ensuite, et ceci est plus curieux, les déviations sont différentes suivant la direction du champ. Il serait évidemment bien prématuré d'attribuer à la magnétite, à la suite de cette observation isolée, une symétrie hémimorphe dans le genre de celle de la tourmaline. Une erreur expérimentale passée inaperçue paraît plus probable, d'autant plus que les expériences sur la magnétite sont bien moins régulières que celles sur les métaux. Les observations correspondant aux directions + et - du champ ont donc été données séparément dans le tableau suivant.

Tableau VIII. - Magnétite $15^{\circ}, 8 \mathrm{C}$.

Zéro observé $=$ Zéro calculé $+0,9 \mathrm{~cm}$.

$\begin{array}{rcc}\text { H gauss } & \begin{array}{c}\text { champ } \\ \text { I2 cm. de l'échelle }\end{array} & \begin{array}{c}\text { champ. } \\ \text { I2 cm. de l'échelie }\end{array} \\ 8600 & 71,49 & 71,72 \\ 18100 & 71,83 & 72,00 \\ 21800 & 71,95 & 72,57 \\ 23300 & 71,99 & 72,57 \\ 24200 & 71,77 & 72,45\end{array}$

$\mathrm{H}$ pression atmospherique, $t=20^{\circ}, 3 \mathrm{~K}$.

$\begin{array}{rrr}8600 & 79, \pi 8 & 79,88 \\ 18100 & 80,69 & 80,79 \\ 21800 & 80,73 & 80,96 \\ 23300 & 80,34 & 81,10 \\ 24200 & 80,08 & 81,37\end{array}$

H solidification, $t=14^{\circ}, 0 \mathrm{~K}$.

$\begin{array}{lll}18100 & 80,90 & 81,10 \\ 21800 & 81,12 & 81,64 \\ 24200 & 80,96 & 81,84\end{array}$

On déduit de ces nombres pour le rapport des intensités d'aimantation à $20^{\circ}, 3 \mathrm{~K}$. et à $15^{\circ}, 8 \mathrm{C}$. :

TABle.1U IX.

$\begin{array}{rrr}\text { H gauss } & \text { Champ }+ & \text { Champ - } \\ 8600 & 1,0559 & 1,0553 \\ 18100 & 1,0591 & 1,0601 \\ 21800 & 1,0593 & 1,0567 \\ 23300 & 1,0504 & 1,0572 \\ 24200 & 1,0563 & \\ \text { Moyenne..... } & 1,0574 & \end{array}$


Donc :

$$
\frac{\mathrm{I}_{200,3 \mathrm{~K}}}{\mathrm{I}_{1 ; 0,8 \mathrm{C}}}=1,0369
$$

non corrigé de la dilatation thermique.

On trouve de même pour le rapport des intensités d'aimantation à $14^{\circ}, 0 \mathrm{~K}$. et $15^{\circ}, 8 \mathrm{C}$. :

done :

$$
1,0609, \quad 1,0622
$$

$$
\frac{\mathrm{I}_{140.0 \mathrm{~K}}}{\mathrm{I}_{130,8 \mathrm{G} .}}=1,0616
$$

rapport s'écartant du précédent dans le sens prévu.

En résumé on a obtenu dans cette partie du travạil concernant les ferromagnétiques, la correction de dilatation thermique n'étant pas faite (voir p. 566 , note 2 ) pour :

$$
\begin{array}{ll}
\text { le nickel } \ldots \ldots \ldots \ldots \ldots & \frac{\mathrm{I}_{200,3 \mathrm{~K} .}}{\mathrm{I}_{170,3 \mathrm{G} .}}=1,0548, \\
\text { le fer } \ldots \ldots \ldots \ldots \ldots & \frac{\mathrm{I}_{200,3 \mathrm{~K} .}}{\mathrm{I}_{20<\mathrm{G} .}}=1,0210, \\
\text { la magnétite........... } & \frac{\mathrm{I}_{200,3 \mathrm{~K} .}}{\mathrm{I}_{150,8 \mathrm{G} .}}=1,0569 .
\end{array}
$$

